\title{
Dwarf Galaxies of the Local Group
}

\author{
Rosanne Di Stefano ${ }^{1,2}$, Roberto Soria ${ }^{1}$, F.A. Primini ${ }^{1}$, \\ and Albert Kong ${ }^{3}$ \\ ${ }^{1}$ Harvard-Smithsonian Center for Astrophysics, 60 Garden St., Cambridge, MA 02138, USA \\ email: rd@cfa.harvard.edu \\ ${ }^{2}$ Department of Physics and Astronomy, Tufts University, Medford, MA 02155 \\ ${ }^{3}$ Center for Space Research, MIT, Cambridge, MA 02139, USA
}

\begin{abstract}
X M M$-Newton and Chandra have ushered in a new era for the study of dwarf galaxies in the Local Group. We provide an overview of the opportunities, challenges, and some early results. The large number of background sources relative to galaxy sources is a major theme. Despite this challenge, the identification of counterparts has been possible, providing hints that the same mechanisms producing X-ray sources in larger galaxies are active in dwarf galaxies. A supersoft X-ray source within $2^{\prime \prime}$ of the supermassive black hole in M32 may be a remnant of the tidal disruption of a giant, although other explanations cannot be ruled out.
\end{abstract}

\section{Introduction}

Dwarf galaxies constitute the largest number of galaxies residing in groups and clusters. Dwarf galaxies also inhabit the field. They are the building blocks of galaxy formation. Therefore, in the young universe and throughout vast volumes of the present-day universe, dwarf galaxies provide the typical environments for the formation and evolution of X-ray sources (XRSs).

A vast array of multiwavelength observations of Local Group dwarf galaxies allow the detailed star formation histories of individual galaxies to be derived. This is important, because theoretical considerations and observations of larger, more distant galaxies suggest that there are correlations between star formation history and the formation of XRSs (see, e.g., Gilfanov 2004, Gilfanov, Grimm, \& Sunyaev 2004, and references therein). There is, e.g., a strong correlation between star formation rates and the formation of high-mass X-ray binaries (HMXBs) and supernova remnants (SNRs). There is also a correlation between low-mass X-ray binaries (LMXBs) and the total galaxy mass; the LMXB population in a large galaxy provides a measure of the average star formation history. Detailed information about individual dwarf galaxies can therefore help to both test and refine theory. The Local Group dwarf galaxies in effect form a set of individual laboratories in which the foundations of X-ray astronomy can be studied.

We can best study X-rays from those dwarf galaxies in the Local Group, where our observations can discover sources of relatively low luminosity, and where we can determine source positions well enough to identify counterparts at other wavelengths. Chandra and XMM-Newton provide new tools. Each can resolve individual systems in the dwarf galaxies of the Local Group, where the X-ray populations are generally not spatially concentrated. The large effective area of XMM-Newton collects enough photons to study many spectral and timing characteristics without prohibitively long exposure times. The superb angular resolution of Chandra allows more reliable detection of counterparts, especially in combination with high resolution optical or radio observations; in some cases, structures in extended sources, such as SNRs, may be resolved. 
Table 1. Source Counts for 4 Galaxies Observed with XMM-Newton

\begin{tabular}{lccccc}
\hline Galaxy & $\begin{array}{c}n_{\mathrm{H}} \\
\left(10^{20} \mathrm{~cm}^{-2}\right)\end{array}$ & $\begin{array}{c}\text { Distance } \\
(\mathrm{Mpc})\end{array}$ & $\begin{array}{c}\text { pn exp. time } \\
(\mathrm{ks})\end{array}$ & $\begin{array}{r}\text { MOS exp. time } \\
(\mathrm{ks})\end{array}$ & $\begin{array}{c}\text { Number of sources } \\
\text { in EPIC field }\end{array}$ \\
\hline NGC 205 & 6.7 & 0.72 & 13.1 & 14.6 & 60 \\
Sextans B & 2.7 & 1.3 & 14.4 & 16.1 & 56 \\
NGC 147 & 12.0 & 0.75 & 10.8 & 12.4 & 53 \\
WLM & 2.0 & 1.0 & 6.7 & 9.2 & 38 \\
\hline
\end{tabular}

\section{Expected Numbers of Sources: The Background Dominates}

The Local Group consists of the Milky Way, M31, M33, and more than 30 dwarf galaxies (see Mateo 1998; van den Bergh 2000). Dwarf galaxy masses range from $<10^{8} M_{\odot}$ to $\sim 10^{10} M_{\odot}$. Morphological types include dwarf spheroidals, irregular galaxies of various shapes, and one elliptical. Central surface densities range from $<11.6$ to $>26$ in mag $\operatorname{arcsec}^{-2}$, with most values lying between 20 and 26. Some dwarf galaxies are close enough to either M31 (e.g., M32 and NGC 205) or the Milky Way (e.g., the Sagittarius dwarf galaxy) to experience tidal interactions. Because of their proximity to Earth, the Magellanic Clouds have been well-studied at X-ray as well as at other wavelengths. In this presentation we will focus on the other dwarf galaxies. These are on the frontiers of X-ray astronomy, and present challenges that can be met by observations with the present generation of $\mathrm{X}$-ray telescopes and with the next generation.

Despite differences among the galaxies, we can predict the number of XRSs expected if the formation of XRSs proceeds in dwarfs much as it does in larger galaxies. Because it is not clear that the correlations we use are correct for masses as small as those of individual dwarf galaxies, we make predictions only for a set of galaxies: M32, NGC 205, IC-10, WLM, NGC 147, Sextans B, the Sagittarius dwarf galaxy. These 7 galaxies represent a range of morphologies, star-formation rates, and star-formation histories. Some are near one of the large Local Group galaxies, and some are relatively isolated. In this set of galaxies we expect to find 2-10 globular cluster XRSs; 6-30 LMXBs, 2-10 HMXBs and SNRs. That is, we predict a total of 10-50 sources with X-ray luminosities near or above $10^{36} \mathrm{erg} \mathrm{s}^{-1}$. (Details will be presented in Di Stefano et al. 2005.)

Foreground and Background Sources: Based on deep field studies, we expect there to be roughly 400 XRSs per square degree with flux $(0.5-2 \mathrm{keV})$ greater than $2 \times 10^{-15}$ erg s${ }^{-1} \mathrm{~cm}^{-2}$ (see, e.g., Giacconi et al. 2002 and references therein). One circular field of radius $13^{\prime}$, which roughly mimics $X M M$-Newton coverage, should therefore contain approximately 60 background sources.

Comparisons: Table 1 shows the results of XMM-Newton observations for 4 galaxies. In each case, the total number of XRSs detected is comparable to the number of background sources expected. The impression that the background plays the dominant role is reinforced by the fact that the overall spatial distributions appear to be more-or-less uniform, with little or no clustering obviously associated with galaxy features. This is illustrated in Figure 1, which shows the sources detected by XMM-Newton in the vicinity of NGC 205, a dwarf galaxy very close to M31. A small fraction of the detected XRSs are associated with the galaxy or its environment. Note, e.g., that one of the sources in Figure 1 is associated with a globular cluster (GC). To determine which other sources may also be associated with the NCG 205 or its environs, IDs with objects at other wavelengths are crucial. The same is true for other dwarf galaxies. 


\section{Individual Sources}

\subsection{M32: Skirting a Supermassive Black Hole}

M32 is the only Local Group dwarf galaxy known to house a massive $\left(\sim 2 \times 10^{6} M_{\odot}\right)$ black hole (BH). There is a clear excess of sources in the vicinity of the BH: 3 within a few arcseconds of the nucleus, while the average density is roughly 1 per 10 square arcminutes (Ho et al. 2003). It is highly likely that the sources near the center are there because of some activity related to the presence of the $\mathrm{BH}$. Indeed, stellar interaction in the dense region around the $\mathrm{BH}$ can produce XRSs.

It is especially interesting that one of the sources within $2^{\prime \prime}$ of the nucleus is a supersoft source (SSS). SSSs have luminosities above $10^{36} \mathrm{erg} \mathrm{s}^{-1}$ and emit little, if any radiation above $1-1.5 \mathrm{keV}$. Nine SSSs located in the Galaxy or in Magellanic Clouds are associated with hot white dwarfs (WDs), and accreting WD models provide promising explanations for the other local SSSs. But interactions in dense stellar environments do not appear to favor the formation of SSS WD binaries based on theoretical arguments and on observations of GCs. Outside of GCs in M31 (which contain no SSSs), SSSs constitute roughly $10 \%$ of all observed XRSs (Di Stefano et al. 2004).

The only other galaxy in the Local Group in which close associations between a supermassive BH and SSSs can be detected is in M31, where there is also a SSS within $2^{\prime \prime}$ of the central BH. That observation inspired the conjecture the tidal stripping of giants by a massive $\mathrm{BH}$ can leave behind a core which will be bright in soft X-rays for an interval of $10^{3}-10^{6}$ years (DiStefano et al. 2001). The M32 observations provide an independent venue in which to test this conjecture and other possibilities as well.

\subsection{The Globular Cluster XRSs of NGC 205 and Sagittarius}

The X-ray luminosity function for Galactic GC XRSs is bimodal, consisting of "bright" sources $\left(L_{x}>10^{36} \mathrm{erg} \mathrm{s}^{-1}\right)$ and "dim" sources $\left(L_{x}<10^{34} \mathrm{erg} \mathrm{s}^{-1}\right)$. The bright sources are LMXBs with neutron star accretors. Even relatively short observations (>10 ksec) with XMM-Newton or Chandra will detect such sources in most of the Local Group dwarf galaxies. The dim sources are either CVs or LMXBs in quiescence. They can only be detected in nearby dwarfs, such as the Sagittarius dwarf galaxy. The results sketched below indicate that the luminosity distribution of XRSs in dwarf galaxy GCs is likely to be similar to that already studied in the Galaxy. The discovery of additional XRSs in the GCs of dwarf galaxies will allow more detailed comparisons to be made. NGC 205: Like M32, NGC 205 is close to M31. Soria et al. (2005) have discovered an XRS associated with one of the GCs, B024, in the field of the galaxy. (See Figure 1; Galleti et al 2004). The Sagittarius Dwarf Galaxy is the nearest dwarf galaxy to us. Ramsay \& Wu (2005) have discovered 7 dim XRSs within the half-mass radius of M54, one of the GCs that is near Sagittarius and that also has a radial velocity consistent with membership in the Sagittarius dwarf galaxy system.

\subsection{Dwarf Galaxies with Active Star Formation}

HMXBs and X-ray active SNRs are expected only in galaxies which have experienced star formation within the past $10^{6}-10^{8}$ years. IC 10 and NGC 6822 both have active star formation. Below we focus on two XRSs for which there is a wealth of supporting evidence linking each to its galaxy. NGC $\mathbf{6 8 2 2}$ contains an XRS near its center, long suspected to be associated with a SNR. The combination of Chandra observations, emission line studies by the Local Group Survey (LGS), and radio observations have made this identification secure. In fact, the SNR is resolved (see Figure 4), and the structures detected in X-ray, radio, and optical wavelengths coincide with each other. (See Kong et al. 2004 for details and additional references.) IC 10 contains an XRS with $L_{X}(0.3-8 \mathrm{keV})=1.2 \times 10^{38} \mathrm{erg} \mathrm{s}^{-1}$. 
This source has been associated with a Wolf Rayet star, indicating that it is a high-mass $\mathrm{X}$-ray binary. Variations by a factor of as much as 6 have been observed to occur over time intervals as short as $10^{4}$ seconds. The spectrum has been fit with a multicolor disk model with $T_{i n}=1.1 \mathrm{keV}$. (See Wang, Whittaker, \& Williams [2005] for details; see also Bauer \& Brandt [2004] and references therein, and Brandt et al. [1997].) These timing and spectral properties are consistent, but are not unique to what we expect from an accreting BH. This interesting system is clearly worthy of further study.

\section{What We Have Learned, What We Can Learn}

Most XRSs found in the vicinity of Local Group dwarf galaxies are associated with background objects. Nevertheless, previous and ongoing studies have discovered several intriguing bright sources with clearly associated counterparts in dwarf galaxies. Additional work should be able to identify galaxy counterparts for several times as many XRSs. Statistical tests can also be helpful. Although they cannot identify individual galaxy sources, they can help to quantify the fraction of observed sources associated with the galaxies by quantifying the level of deviations from spatial uniformity, or deviations from the luminosity function expected for the background.

The primary result derived so far is that the mechanisms that produce XRSs in dwarf galaxies appear to be those already well-studied in other, larger galaxies. This is important to know if we are to estimate the X-ray source contribution from dwarf galaxies in other parts of the Universe.

Refined predictions of the XRS population for individual dwarf galaxies, based on the star formation history inferred from multiwavelength studies, are needed. X-ray observations can then test the predictions, and possibly discover new effects. One effect already discovered in both M31 and M32 is the proximity of an SSSs to a massive BH. These SSSs could be remnants of giants that have been tidally stripped by the BH. Additional work is needed to determine is this is likely, or if other scenarios are preferred.

Together, XMM-Newton and Chandra make a good team for conducting studies of Local Group dwarf galaxies. The immediate scientific returns are large. In addition, ongoing observations can play an important role in preparing the way for the next generation of $\mathrm{X}$-ray telescopes. The new telescopes will have significantly larger effective areas, while spatial resolution may not be quite as good as presently available. A modest decline in spatial resolution will not hobble future studies of Local Group dwarf galaxies, because the distribution of most sources is not spatially concentrated. On the other hand, larger effective area will provide spectral and timing information that can help us to better understand the physical nature of the sources.

\section{References}

Bauer, F. E. \& Brandt, W. N. 2004, ApJL, 601, L67

Brandt, W. N., Ward, M. J., Fabian, A. C., \& Hodge, P. W. 1997, MNRAS, 291, 709

Di Stefano, R., et al. 2004, ApJ, 610, 247

Di Stefano, R., Greiner, J., Murray, S., \& Garcia, M. 2001, ApJL, 551, L37

Galleti, S., Federici, L., Bellazzini, M., Fusi Pecci, F., \& Macrina, S. 2004, A\&A, 416, 917

Giacconi, R., et al. 2002, ApJS, 139, 369

Gilfanov, M., Grimm, H.-J., \& Sunyaev, R. 2004, MNRAS, 347, L57

Gilfanov, M. 2004, MNRAS, 349, 146

Ho, L. C., Terashima, Y., \& Ulvestad, J. S. 2003, ApJ, 589, 783

Kong, A. K. H., Sjouwerman, L. O., \& Williams, B. F. 2004, AJ, 128, 2783

Mateo, M. L. 1998, ARA\&A, 36, 435

Ramsay, G. \& Wu, K. 2005 (private communication).

van den Bergh, S. 2000, The galaxies of the Local Group, ISBN: 0521651816.

Wang, Q. D., Whitaker, K. E., \& Williams, R. 2005, MNRAS, 362, 1065 


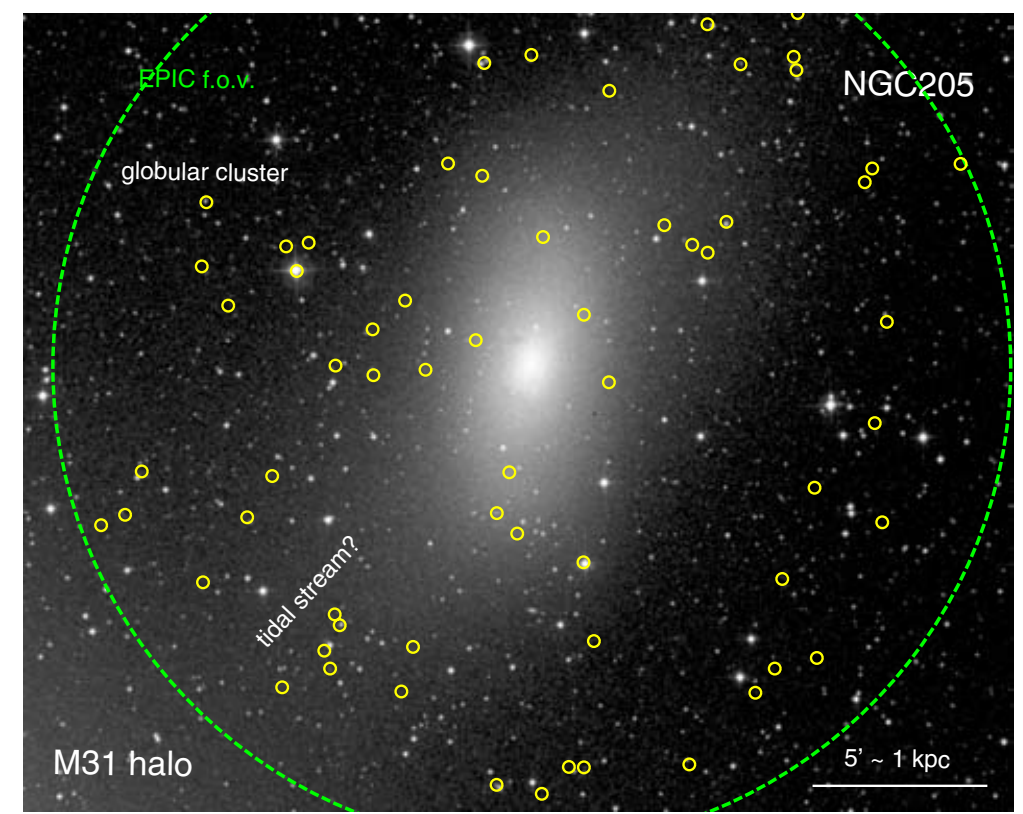

Figure 1. NGC205 XRSs (yellow open circles) detected by XMM-Newton, overlaid on an optical image. The position of one XRS coincides with the position of a globular cluster.

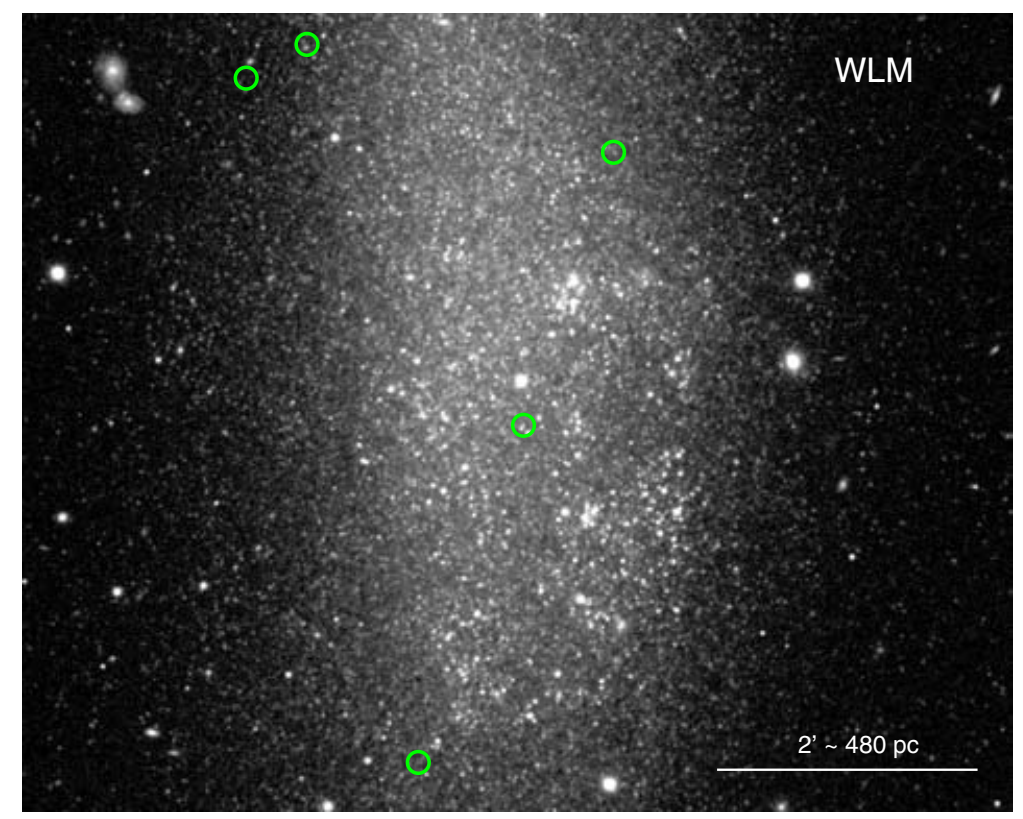

Figure 2. XRSs (green open circles) detected by XMM-Newton, overlaid on an optical image of WLM from the Local Group Survey. 


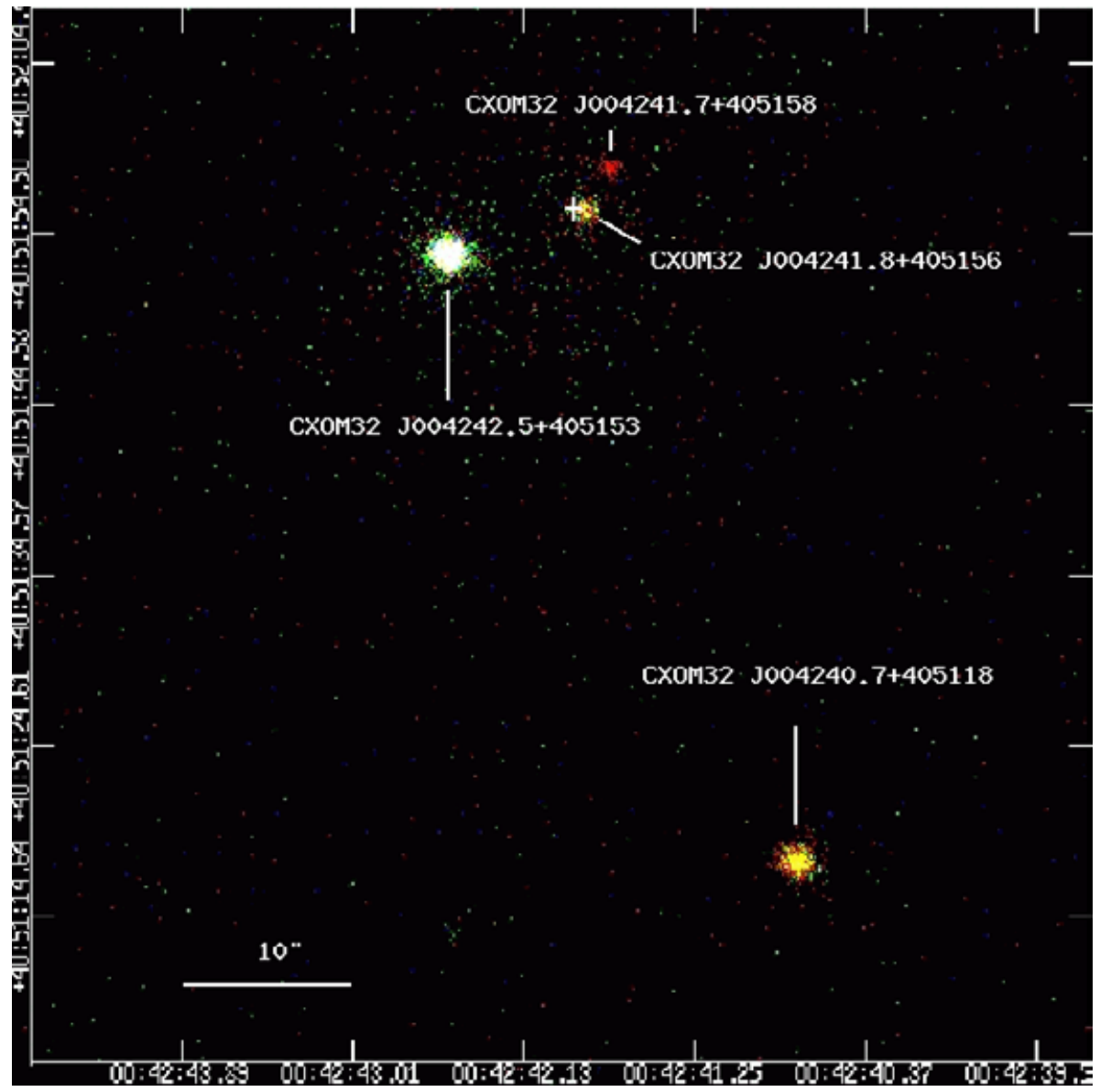

Figure 3. M32: The white cross marks the galaxy center; the red source is supersoft (the NW-most source, J004241.7+405158).
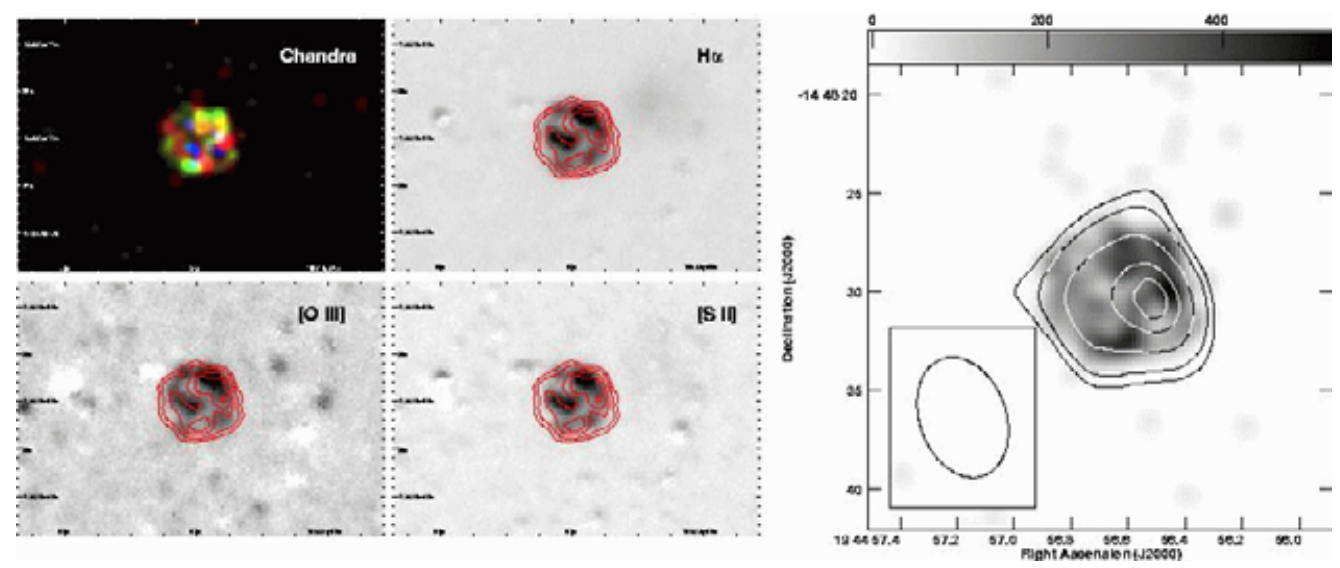

Figure 4. SNR near the center of NGC 6822. Right: radio contours superposed on the Chandra image. Left: upper left panel shows the Chandra image. Proceeding clockwise, the Chandra contours are superposed on the $\mathrm{H}_{\alpha}$, [S II], and [O III] images. 